\title{
Beobachtungen des Cometen 1888 I
}

angestellt auf der Sternwarte in Kremsmunster.

\begin{tabular}{|c|c|c|c|c|c|c|c|c|c|c|c|c|c|c|c|c|c|}
\hline \multicolumn{2}{|c|}{1888} & \multicolumn{2}{|c|}{ M.Z.Kr. } & \multicolumn{2}{|r|}{$\Delta \alpha$} & \multicolumn{2}{|r|}{$\Delta \delta$} & gl. & \multicolumn{3}{|c|}{$\alpha$ app. } & $\operatorname{og} p .4$ & \multicolumn{2}{|c|}{$\delta$ app. } & $\operatorname{og} p \cdot \Delta$ & Red. ad 1. app. & * \\
\hline & & $6^{\text {h }} 4$ & $8^{m} 5^{3}$ & $-0^{x}$ & & + & $5^{\prime} 5^{8.1}$ & 9 & & & & $9.5^{6} 5 n$ & $+0^{\circ} 2$ & & 815 & $-1: \ln -7: 0$ & \\
\hline & 7 & 1627 & 7. 33 & -0 & & + & 19.7 & 6 & 22 & 21 & & & +955 & 8.5 & .788 & $-0.93-8.3$ & \\
\hline & 15 & $155^{8}$ & 811 & +2 & & + & & 6 & 22 & 44 & & & +1646 & & & $-0.82-9.6$ & \\
\hline & 16 & 1553 & 5331 & +2 & 32 & 一 & I 39.0 & 5 & 22 & 47 & & $9.607 n$ & +1732 & & & $-0.81-9.7$ & \\
\hline & 17 & 1526 & 631 & -0 & & + & $0 \quad 14.7$ & 6 & 22 & 49 & & & +1816 & & & $-0.81-9.8$ & \\
\hline & 22 & 15 & 116 & -0 & & 一 & $4 \quad 15.5$ & 10 & & 3 & & 9.6 & +2147 & & 760 & $-0.75-10.4$ & 6 \\
\hline & 23 & 1521 & 128 & +3 & & + & I 11.4 & 6 & & & & & +2226 & & & $-0.72-10.5$ & 7 \\
\hline & 24 & 1520 & $0 \quad 3^{8}$ & -1 & & -1 & 044.5 & 6 & 23 & 8 & & $9.630_{n}$ & +234 & & & $-0.73-$ & \\
\hline & 29 & 1446 & 625 & +2 & & + & $0 \quad 48.3$ & 6 & 23 & 20 & & & +26 & & & $-0.69-$ & 9 \\
\hline & 2 & 1420 & 050 & +1 & & 一 & 424.2 & 6 & & 28 & & & +2742 & & & $-0.60-1 \mathrm{I} .3$ & 10 \\
\hline & 13 & $134^{8}$ & 837 & -0 & & - & 88.9 & 6 & 23 & $5^{2}$ & & & +3259 & & & $-0.44-1 \mathrm{r} .9$ & $\mathbf{r}$ \\
\hline & 13 & 1415 & 520 & +2 & & + & 813.0 & 6 & 2 & 52 & & $9.673 n$ & $\vdash 32 \quad 59$ & & & 11.8 & 2 \\
\hline & 15 & 1359 & 922 & +1 & & + & 48.2 & 6 & 23 & 56 & & $9.676_{n}$ & -3350 & .1 & & $-0.40-1 \mathrm{x} .8$ & 3 \\
\hline & 16 & 1429 & 4 & +2 & I.74 & + & 241.5 & 6 & 23 & $5^{8}$ & & & $+34: 5$ & 2.4 & & $-0.37-12.0$ & 4 \\
\hline & 17 & $141 \%$ & 759 & -3 & & - & 057.5 & 6 & & 0 & & 9.6 & +3439 & & & I 2.0 & 5 \\
\hline & 22 & 14 & 851 & +1 & & + & 419.9 & 6 & & 10 & & & +3635 & & & $-0.26-$ & 6 \\
\hline & 24 & 13 & 939 & -1 & & - & r $\quad 3.6$ & 6 & o & 13 & & 9.6 & +3719 & & & $-0.23-12.2$ & 17 \\
\hline & 27 & 1422 & 215 & $-\mathbf{I}$ & & + & 354.7 & 8 & 0 & 19 & & & +3823 & & & -0. & 18 \\
\hline Juni & I & 149 & 28 & -4 & 0.48 & 一 & $3 \quad 32.5$ & 6 & 0 & 27 & 12 & $9.710_{n}$ & +402 & & & $-0.06-12.3$ & 9 \\
\hline & 2 & 1424 & 423 & +4 & & 一 & - 38.4 & 6 & 0 & 28 & & 9.7 & +4022 & & & $+0.02-12.2$ & 20 \\
\hline & 7 & & 554 & -2 & & + & I 4.1 & 6 & 0 & 36 & I I & $9.7 \times 9 n$ & +4553 & & & $0-12.2$ & $2 \mathbf{I}$ \\
\hline & 8 & 1350 & - 33 & +1 & & 一 & I $\mathbf{3 2 . 3}$ & 6 & $\circ$ & 37 & & $9.725 \mathrm{n}$ & +42 II & & & $+0.16-12.2$ & 22 \\
\hline & 12 & 1344 & 421 & -2 & & + & - 28.1 & 6 & $\circ$ & 43 & & 9.7 & +4319 & & & +0.25 & 23 \\
\hline & & 1344 & 45 & $-\mathbf{I}$ & & + & 048.7 & 6 & ○ & 44 & & & +4335 & & & $+0.28-12.1$ & 24 \\
\hline & $\mathbf{I}$ & 1359 & 928 & +0 & 24.0 & 一 & - 6.0 & 6 & 0 & 46 & & & +449 & & & $+0.38-12.1$ & 25 \\
\hline & & 1358 & $84 I$ & +0 & & $T$ & I 11.8 & 6 & $\circ$ & 47 & & & +4425 & & & $+0.39-12.1$ & 26 \\
\hline & 9 & 1328 & 82 & +0 & & & 132.4 & 2 & $\mathbf{T}$ & & & 9.74 & +4946 & 16.9 & 0.379 & $+1.24-10.7$ & 27 \\
\hline
\end{tabular}

Beobachter: Prof. F. Schwab.

Mittlere Oerter der Vergleichsterne für 1888.0.

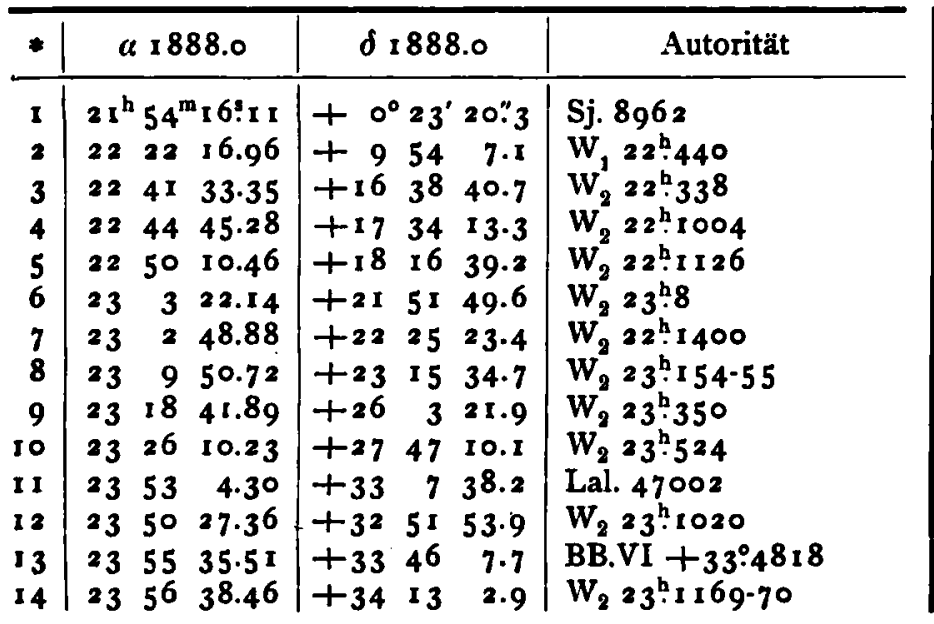

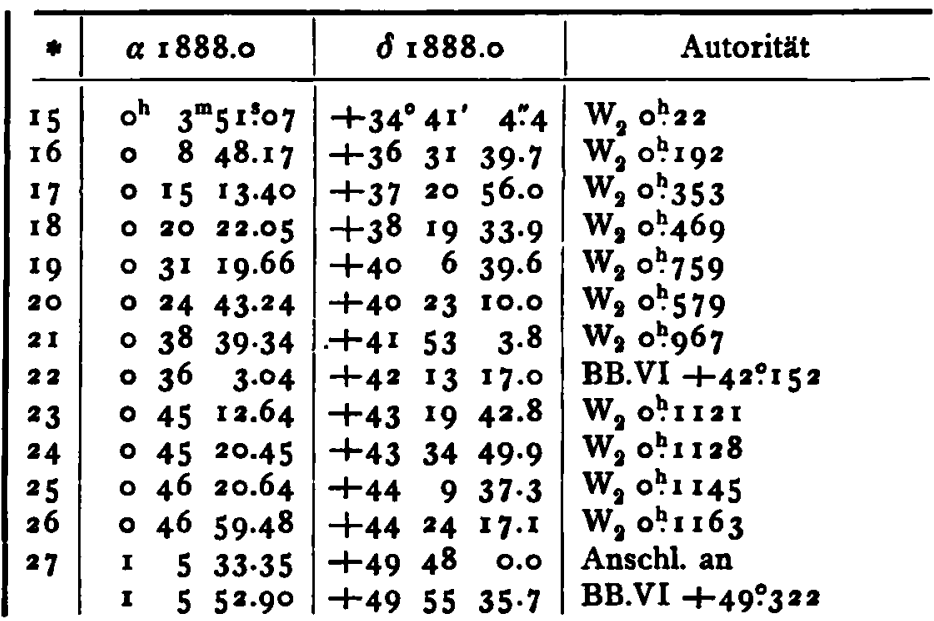

Bemerkungen.

März 29. Kopf und Schweif sehr hell, letzterer etwa 16 $6^{\circ}$ April 15 . Der Schweif reicht bis zum Vergleichstern und ist lang, schmal, in westlicher Richtung vom Kern, vom Parallel wenig gegen Süden abweichend.

April 7. Schweif im Sucher $1^{\circ}$ lang. auf der Südseite gegen den Kern hin heller als auf der Nor dseite.

2 22. Die Schweiflange bei Mondschein 16'. 
April 24. Wegen Wolkenschleier und Vollmond der Comet schwach sichtbar.

2 29. Der Schweif etwa 20' lang, schwach gekrummt, mit der convexen Seite nach Norden.

Mai 13. Ausdehnung des Cometen ziemlich unverändert. Die Austritte sind, da der Schweif vorangeht und ganz allmählich in den verwaschenen Kern ubergeht, am Ringmikrometer schwierig aufzufassen.

- I 7. Comet noch in der früheren Ausdehnung sichtbar, Kern sehr schwach.

- 22. Der Kopf trotz des Mondscheins auffallend hell, vom Schweif kaum eine Spur zu sehen, dagegen nach Norden und Süden vom Kopfe schwach sichelförmig nach Westen gekrümmite Lichtausstrahlungen. Aehnlich ist das Aussehen auch am 24. und 27.
Juni I. Ausser den 2 vom Kopfe ausgehenden Lichtbögen ist auch der gewöhnliche Schweif wieder in einer Länge von $20^{\circ} \mathrm{zu}$ sehen.

- 8. Der Kopf des Cometen ist noch etwas verbreitert, Schweif 15 ' lang.

12. Von den Lichtausstrahlungen senkrecht zur Richtung der Bewegung ist nichts mehr wahrzunehmen. Das erste Viertel des Schweifes ist verhältnissmässig hell.

- I 5. Aussehen wenig verändert. Kern eben noch sichtbar.

29. Nur zeitweilig ein Kernchen zu bemerken.

Juli 9. Der Comet ist nur mehr als ein etwa $10^{\circ}$ langer schmaler Nebelstreifen von fast gleichmässiger Helligkeit zu sehen. Auffassung schwierig. Fortsetzung der Beobachtung durch Wolken verhindert.

Kremsmünster 1888 Oct. 21 . Col. IVagner.

\section{Observations of Comet 1888 I}

made with the 15 inch. Equatorial of the Harvard College Observatory by O.C. Wendell, Assistant. (Communicated by Professor Edward C. Pickering, Director.)

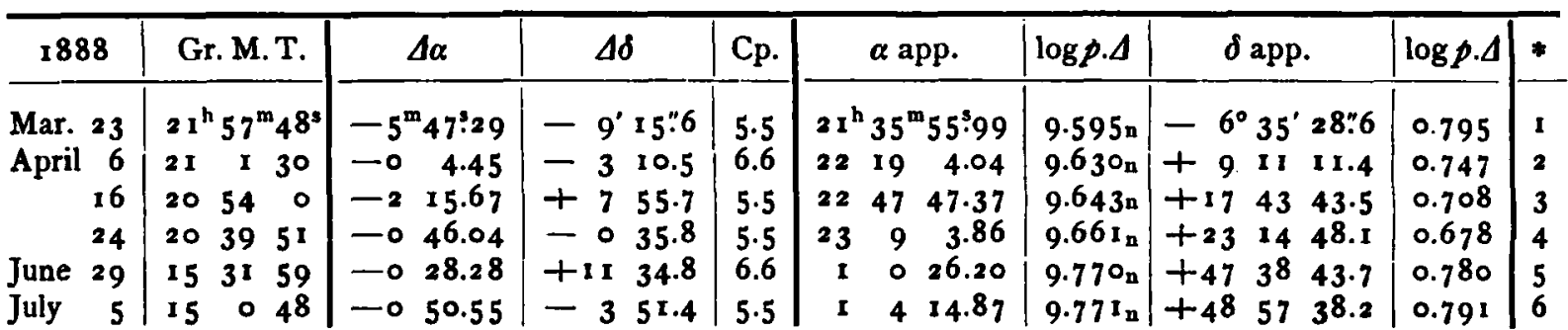

Mean Places of the Comparison Stars for 1888.0 .

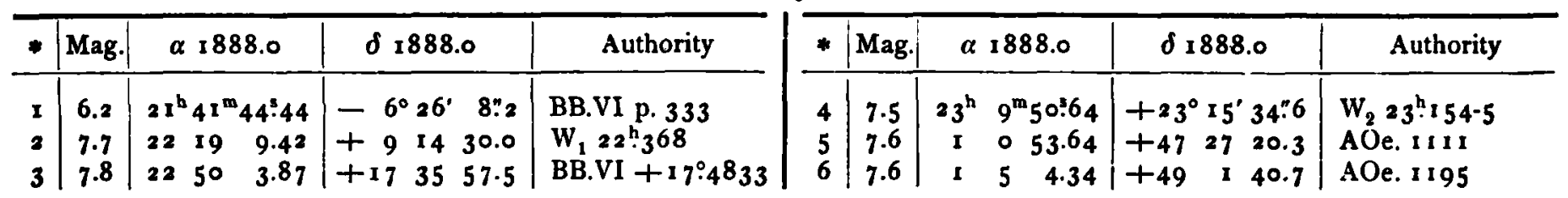

Osservazioni della cometa 1888 III (Brooks Aug. 7)

fatte all'equatoriale di $25^{\mathrm{cm}}$ di apertura del R. Osservatorio del Coll. Romano da E. Millosevich. (Vedi A. N. 2855-56.)

\begin{tabular}{|c|c|c|c|c|c|c|c|c|c|}
\hline 1888 & T.M.Roma & $\Delta a$ & $\Delta \delta$ & Cfr. & $\alpha$ app. & $\operatorname{og} p .4$ & $\delta$ app. & $\log p . \Delta$ & \\
\hline & & $\begin{array}{l}+0^{m} 7^{5.27} \\
-120.80\end{array}$ & & $\begin{array}{l}6.6 \\
8.8\end{array}$ & $12^{\mathrm{h}} 4^{6^{\mathrm{m}}} 5$ & & & & \\
\hline Sett. & $\begin{array}{lll}8 & 28 & 25\end{array}$ & $\begin{array}{r}\text { to } 21.99 \\
\text { to }\end{array}$ & 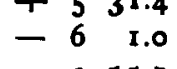 & $\begin{array}{l}8.0 \\
8.7\end{array}$ & $\begin{array}{rrr}15 & 19 & 42.74\end{array}$ & & $\begin{array}{r}+375737.5 \\
+165853.0\end{array}$ & $\begin{array}{l}0.707 \\
0.734\end{array}$ & 3 \\
\hline Ott. & 7750 & $-.0 \quad 17.79$ & -355.7 & 9.9 & $15 \quad 36 \quad 27.64$ & 9.612 & $1+133^{8} 13.2$ & 0.703 & \\
\hline
\end{tabular}

Stelle di confronto.

\begin{tabular}{|c|c|c|c|}
\hline$*$ & $\alpha 1888.0$ & $\delta 1888.0$ & Autorità \\
\hline $\begin{array}{l}\text { I } \\
\text { 2 }\end{array}$ & 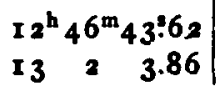 & $\left|\begin{array}{rrrr}+39^{\circ} & 9^{\prime} & 21.8 \\
+37 & 51 & 59.0\end{array}\right|$ & $\begin{array}{l}\text { DM. }+39^{\circ} 2572 \text { rif. a } W_{2} 935 \\
\text { Rad. } 2964\end{array}$ \\
\hline
\end{tabular}

\begin{tabular}{|c|c|c|c|}
\hline$*$ & $\alpha 1888.0$ & $\delta 1888.0$ & Autorità \\
\hline $\begin{array}{l}3 \\
4\end{array}$ & $\begin{array}{l}15^{h} 19^{m} 20^{8}: 5^{6} \\
15 \quad 3^{6} 45.17\end{array}$ & 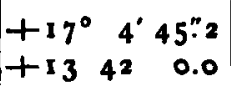 & $\begin{array}{l}W_{2} 5^{h} \cdot 388 \\
D M .+13^{\circ} 2983 \text { rif. a } W_{1} 612\end{array}$ \\
\hline
\end{tabular}

Sett. 27. La cometa si presentava con debole luce. - Ott. 2. La cometa e debole; tuttavia scorgesi ancora un nucleo di $\mathrm{I}^{\mathrm{ma}}$ grandezza. - La sera del 23 Ottobre la cometa si vede solo intermittentemente benchè il cielo sia sereno.

Roma 1888 Ottobre 24. E. Millosevick. 